\title{
X-ray diffuse scattering from stacking faults in thick
}

\section{C-SiC single crystals}

\author{
A. Boulle \\ Science des Procédés Céramiques et de Traitements de Surface CNRS UMR 6638, \\ ENSCI 47 avenue Albert Thomas 87065 Limoges Cedex, France \\ D. Chaussende, L. Latu-Romain \\ Laboratoire des Matériaux et du Génie Physique CNRS UMR 5628, INPGrenoble, \\ BP 46, 38402 Saint-Martin d'Hères Cedex, France \\ F. Conchon, O. Masson, R. Guinebretière \\ Science des Procédés Céramiques et de Traitements de Surface CNRS UMR 6638, \\ ENSCI 47 avenue Albert Thomas 87065 Limoges Cedex, France
}

\begin{abstract}
Stacking faults in thick (001)- and (111)-oriented 3C-SiC single crystals are studied by high resolution X-ray diffraction. We demonstrate that the analysis of the diffuse scattering intensity distribution can be used as a non-destructive means to accurately determine the densities of Shockley type stacking faults. The diffuse scattering intensity is simulated with a scattering model based on a difference-equation description of faulting in fcc materials. It is shown that the (001) $\mathrm{SiC}$ crystals exhibits an anisotropic fault distribution, whereas the (111) SiC crystals exhibit an isotropic fault distribution, in excellent quantitative agreement with transmission electron microscopy observations.
\end{abstract}

PACS codes: 61.72.Nn, 81.05.Hd, 61.72.Dd 
According to outstanding electrical properties, the cubic silicon carbide polytype (3C-SiC) presents some additional interests compared to the hexagonal structures. For example, superior inversion channel mobility is expected in metal-oxide-semiconductor field-effect transistors devices ${ }^{1}$. However, despite decades of 3C-SiC materials studies, expected theoretical performances of electronic devices have never been demonstrated to date, because of the too poor 3C-SiC crystals quality. Even in the best "free-standing" 3C-SiC wafers, the extended defects (mainly stacking faults, SFs) density still is much higher than in the high quality commercial $4 \mathrm{H}$ or $6 \mathrm{H}-\mathrm{SiC}$ wafers ${ }^{2}$. Consequently, most devices fabricated from this material have average performances such as blocking capability to about $100 \mathrm{~V}^{3}$. A preliminary for the development of high performances 3C-SiC based devices is thus the availability of high quality bulk $3 \mathrm{C}$-SiC crystals. Along this line, the potential of the continuous feed physical vapor transport (CF-PVT) process ${ }^{4}$ has been recently demonstrated for the growth of bulk-like 3C-SiC layers ${ }^{5,6}$. The need of a non-destructive tool to investigate both qualitatively and quantitatively the SFs in 3C-SiC crystals is now of primary importance for a further development of the material itself and the associated growth process. That is the topic of the present letter.

So far, SFs in SiC films have been evaluated by X-ray diffraction (XRD) and X-ray topography $(\mathrm{XRT})^{7}$, transmission electron microscopy (TEM) $)^{8,9}$, Raman scattering ${ }^{10}$ and photoluminescence $^{11}$. Apart from TEM and XRT, none of these techniques was used quantitatively in the sense that SF densities were not obtained. Moreover, most of these studies dealt with the $4 \mathrm{H}$ and $6 \mathrm{H}$ polytypes. In this letter, we report on high-resolution XRD (HRXRD) experiments performed on thick $3 \mathrm{C}-\mathrm{SiC}$ single crystals. We develop a method of determining the SF density from the simulation of the scattered intensity distribution in reciprocal space. Advantages of XRD are that no sample preparation is necessary and, because of the large X-ray 
beam size (several $\mathrm{mm}$ ) it provides averaged, statistically relevant, measurements of the SF density.

In the present study, we analyze two different $3 \mathrm{C}-\mathrm{SiC}$ single crystals. The first one is a commercially available $250 \mu \mathrm{m}$ - thick (001) oriented crystal grown by chemical vapor deposition (CVD) on undulant Si substrate, from HAST Corporation ${ }^{2}$. The second sample is a $400 \mu \mathrm{m}$ - thick (111) oriented crystal which has been grown on a $30 \mathrm{~mm}$ - diameter (0001) 4HSiC substrate in a CF-PVT crucible. Both CF-PVT process and growth parameters are detailed elsewhere ${ }^{5}$. HRXRD experiments were conducted on a laboratory equipment mounted on a high power X-ray source and equipped with a curved position sensitive detector (PSD) $)^{12}$. Maps of the diffracted intensity close to the (002) Bragg peak of (001) SiC and close to the (111) Bragg peak of (111) SiC were recorded for different azimuthal positions of the sample (Fig. 1).

All intensity distributions exhibit common features. The very weak streak parallel to the normal to the surface (i.e. parallel to [001] for (001) $\mathrm{SiC}$ and parallel to [111] for (111) $\mathrm{SiC}$ ) is due to the truncation of the crystal lattice at the surface, the so-called crystal truncation rod $(\mathrm{CTR})^{13}$. The intense streak labeled "PSD" lying along the Ewald sphere is due to the transmittance function of the PSD $^{12}$. In Fig. 1(a) and (b) the measured angle between the two remaining streaks and the surface normal is $\psi \approx 55^{\circ}$ which corresponds to the angle between the $\{111\}$ planes and the crystal surface $\left(\psi_{(111),(001)}=54.74^{\circ}\right)$. These streaks of scattered intensity can hence be attributed to SFs lying in the $\{111\}$ planes. Similar diffuse scattering streaks caused by $\{111\}$ SFs have been encountered in Si single crystals ${ }^{14}$. In Fig. 1(c), the streak with angle $\psi \approx 70^{\circ}$ is due to SFs lying in the $(-111)$ plane $\left(\psi_{(111),(-111)}=70.53^{\circ}\right)$, whereas the streak with angle $\psi \approx 55^{\circ}$ is due to SFs lying in the (11-1) and (1-11) planes. The [11-1] and [1-11] directions are not contained in the detection plane so that in principle the latter streak should not appear, but it 
nonetheless appears because of the lack of resolution perpendicular to the detection plane ${ }^{12}$. Its angle towards [111] is thus given by $\psi=$ atan $\left[\tan \psi_{(111),(-111)} \times \cos \phi_{(-111)}\right]=54.74^{\circ}$, where $\phi_{(-111)}$ is the azimuthal angle between the fault plane considered and the detection plane $\left(\phi_{(-111)}=60^{\circ}\right)$. The same conclusions hold for Fig. 1(d) with SFs lying in the (11-1) plane on the one hand, and SFs lying in the (-111) and (1-11) planes on the other hand. The diffuse scattering streaks are more extended in (111) SiC than in (001) SiC which, hence, suggests a higher SF density. Moreover, in (001) $\mathrm{SiC}$ the diffusely scattered intensity is more pronounced along the [1-10] direction than along [110] suggesting a higher defect density in the (-111) and (1-11) planes than in the (111) and (-1-11) planes.

We now develop a model that allows extracting the SF density from the diffusely scattered intensity distribution. Most elaborated descriptions of diffuse scattering from stacking faults in single crystals have been developed to describe the effects of Frank dislocation loops in FCC metals ${ }^{15}$ and Si crystals ${ }^{14}$, although, even in these cases, the obtaining of SF densities is not straightforward. Such dislocations are caused by point defect coalescence and are hence of limited spatial extension. This is in contrast with SFs in $3 \mathrm{C}-\mathrm{SiC}$ which are deformation faults (Shockley faults) and extend over large distances in the crystal ${ }^{9}$. The description developed for Frank faults can hence not be used. Our approach is based on the difference equation method applied to FCC materials described in details by Warren ${ }^{16}$. Within this formalism, the diffusely scattered intensity distribution perpendicular to the fault plane can be written

$$
I\left(Q_{\langle 111\rangle}\right)=\sum_{m=-\infty}^{\infty} \tilde{R}(m) V(m) G(m) \exp \left(i Q_{\langle 111\rangle} \cdot m \cdot d_{111}\right)
$$

where $\mathrm{d}_{111}$ is the planar spacing of the (111) planes, $Q_{<111>}$ is the component of the scattering vector $\mathbf{Q}$ along a particular $<111>$ direction, $\widetilde{R}$ is the Fourier transform of the resolution function of the diffractometer which can be precisely evaluated ${ }^{12}, V$ is the correlation volume 
and $G$ is the pair correlation function describing the effect of faulting as detailed below. The correlation volume depends on the shape and size (and their fluctuations) of the crystalline domains over which diffraction is coherent (mosaic domains) and can be calculated analytically for many relevant cases ${ }^{17}$. In the present case, the analysis of transverse scans performed through the center of all the investigated reflections (not shown here) revealed that finite size effects can be neglected. In the following the mosaic domains were hence assimilated to cubes with dimensions much larger $(6 \mu \mathrm{m})$ than the diffractometer coherence length. The pair correlation function can be written ${ }^{16}$

$$
G(m)=P_{A A}(m)+P_{A B}(m) \exp \left(-2 \pi i \frac{H-K}{3}\right)+P_{A C}(m) \exp \left(2 \pi i \frac{H-K}{3}\right)
$$

where $H=(k-h) / 2$ and $K=(l-k) / 2$. Equation (2) contains the probabilities $P_{A A}(m), P_{A B}(m)$ and $P_{A C}(m)$ to find two identical layers (i.e. in the classical $A B C$ notation, $A-A, B-B$ or $\left.C-C\right) m$ layers apart, or to find two layers in the regular stacking sequence $(A-B, B-C$ or $C-A) m$ layers apart, or in the reverse sequence $(A-C, C-B$ or $B-A)$, respectively. This method was initially developed to analyze powder samples ${ }^{16}$ in which the stacking sequences $\mathrm{ABC}$ and $\mathrm{ACB}$ are equally probable because of the random orientation of the crystallites, so that for a powder sample $P_{A B}(m)=$ $P_{A C}(m)=\left(1-P_{A A}(m)\right) / 2$. In a single crystal this is no longer true and the probabilities must be derived without this assumption. We obtain

$$
\begin{aligned}
& P_{A A}(m)=\left\{1+2(-1)^{m} Z^{|m|}[\cos m \gamma+(\beta / s) \sin |m| \gamma]\right\} / 3 \\
& P_{A C}(m)=\left(1+(-1)^{m+1} Z^{|m|}\{\cos m \gamma+[(6 \alpha+\beta-3) / s] \sin |m| \gamma\}\right) / 3
\end{aligned}
$$

where $Z=\left(1-3 \alpha-2 \beta+3 \alpha^{2}\right)^{1 / 2}, s=\left(3-12 \alpha-6 \beta-\beta^{2}+12 \alpha^{2}\right)^{1 / 2}, \gamma=\operatorname{atan}[s /(1-\beta)]$ and $P_{A A}(m)$ $+P_{A B}(m)+P_{A C}(m)=1$. The only free parameters in $G$ (and hence in the intensity distribution) 
are $\alpha$ and $\beta$, the deformation faulting and twin faulting probabilities which are related to the deformation and twin fault densities through $\rho_{\mathrm{D}}=\alpha / \mathrm{d}_{111}$ and $\rho_{\mathrm{T}}=\beta / \mathrm{d}_{111}$, respectively.

Equations (1)-(3) are used to analyze the intensity distribution in the diffuse streaks. An important difficulty in the analysis is that $\rho_{\mathrm{D}}$ and $\rho_{\mathrm{T}}$ yield similar effects, i.e. they produce a broadening of the intensity distribution along $Q_{<111>}$ (a detailed description of the model will be given elsewhere). Their respective influence of the intensity distribution can hence hardly be separated using solely XRD data. In the present case TEM observations performed on similar (001) $\mathrm{SiC}$ samples $^{9}$ as well as on the (111) $\mathrm{SiC}$ samples $^{6}$ revealed that they are free of twin faults, $\rho_{\mathrm{T}}$ was hence fixed to 0 . The results are displayed in Fig. 2 . The model (full line) fits the data remarkably well over almost four orders of magnitude. The sensitivity of the diffuse scattering on the SF density is illustrated with dashed curve, which corresponds to the case where no faulting is present: faulting mainly affects the profile tails, whereas the central part of the peak remains unaffected. For (001) $\mathrm{SiC}$ we find $\rho_{\mathrm{D}}=6.4 \times 10^{3} \mathrm{~cm}^{-1}$ along $[1-10]$ and $\rho_{\mathrm{D}}=1.4 \times 10^{3}$ $\mathrm{cm}^{-1}$ along [110], in excellent agreement with the values deduced from TEM observations for the similar samples ${ }^{9}$, namely $\rho_{\mathrm{D}}=6 \times 10^{3} \mathrm{~cm}^{-1}$ along [1-10] and a density 3 to 5 times lower along [110]. For (111) SiC, both streaks could be fitted with $\rho_{\mathrm{D}}=2.2 \times 10^{4} \mathrm{~cm}^{-1}$. The SFs emerging at the surface of the crystal create steps which are responsible for the (roughness-induced) damping of the CTR ${ }^{13}$. This explains why the CTR intensity is weaker in (111) $\mathrm{SiC}$ and along [1-10] in (001) $\mathrm{SiC}$.

In summary, we have proposed a powerful method for the investigation of stacking faults in 3C-SiC single crystals. This method, based on the analysis the X-ray diffuse scattering, is able to provide both qualitative and quantitative results. From the quantitative viewpoint, the SF densities are extracted from the simulation of the scattered intensity distribution along the diffuse 
streaks in reciprocal space. Values are in excellent agreement with those measured by TEM. Finally, this method is non destructive and provides averaged, statistically relevant measurements of the SF density. It could surely help the development of the 3C-SiC material and hence an improvement of the device performances based on crystals with reduced SF density. 


\section{REFERENCES}

2

G. Pensl, M. Bassler, F. Ciobanu, V. Afanas'ev, H. Yano, T. Kimoto, and H. Matsunami, Mater. Res. Soc. Symp. Proc. 640, H3.2.1 (2001).

H. Nagasawa, K. Yagi, T. Kawahara, N. Hatta, G. Pensl, W. J. Choyke, T. Yamada, K. M. Itoh, and A. Schoner, in Silicon Carbide, edited by W.J. Choyke, H. Matsunami, and G. Pensl (Springer, New-York, 2004), pp. 207.

A. Schöner, M. Bakowski, P. Ericsson, H. Strömberg, H. Nagasawa, and M. Abe, Mater. Sci. Forum 483-485, 801 (2005).

D. Chaussende, M. Ucar, L. Auvray, F. Baillet, M. Pons, and R. Madar, Cryst. Growth \& Design 5 (4), 1539 (2005).

D. Chaussende, L. Latu-Romain, L. Auvray, M. Ucar, M. Pons, and R. Madar, Mater. Sci. Forum 483-485, 225 (2005).

L. Latu-Romain, D. Chaussende, C. Balloud, S. Juillaguet, L. Rapenne, E. Pernot, J. Camassel, M. Pons, and R. Madar, in Proceedings of the 11th International Conference on Silicon Carbide and Related Materials (ICSCRM2005), September 19-23 (2005), Pittsburgh, USA (Trans Tech Publication, Ütikon-Zürich, 2006), p. in press.

M. Dudley, X. Huang, and W. M. Vetter, J. Phys. D: Appl. Phys. (10A), A30 (2003); W. M. Vetter and M. Dudley, J. Cryst. Growth 260 (1-2), 201 (2004).

H. J. Chung, J. Q. Liu, and M. Skowronski, Appl. Phys. Lett. 81 (20), 3759 (2002).

E. Polychroniadis, M. Syvajarvi, R. Yakimova, and J. Stoemenos, J. Cryst. Growth 263 (1-4), 68 (2004).

S. Nakashima, Y. Nakatake, H. Harima, M. Katsuno, and N. Ohtani, Appl. Phys. Lett. 77 (22), 3612 (2000).

S. Izumi, H. Tsuchida, I. Kamata, and T. Tawara, Appl. Phys. Lett. 86 (20), 202108 (2005).

A. Boulle, O. Masson, R. Guinebretiere, A. Lecomte, and A. Dauger, J. Appl. Crystallogr. 35 (5), 606 (2002).

I. K. Robinson, Phys. Rev. B 33 (6), 3830 (1986).

U. Beck, T. H. Metzger, J. Peisl, and J. R. Patel, Appl. Phys. Lett. 76 (19), 2698 (2000); D. Luebbert, J. Arthur, M. Sztucki, T. H. Metzger, P. B. Griffin, and J. R. Patel, Appl. Phys. Lett. 81 (17), 3167 (2002); P. Klang, V. Holy, J. Kubena, R. Stoudek, and J. Sik, J. Phys. D: Appl. Phys. (10A), A105 (2005).

B. C. Larson and W. Schmatz, Phys. Rev. B 10 (6), 2307 (1974); B. C. Larson and W. Schmatz, Phys. Stat. Sol. (b) 99, 267 (1980); P. Ehrhart, H. Trinkaus, and B. C. Larson, Phys. Rev. B 25 (2), 834 (1982).

B. E. Warren, X-ray diffraction. (Addison-Wesley, New-York, 1969).

A. Boulle, R. Guinebretière, and A. Dauger, J. Phys. D: Appl. Phys. 38 (21), 3907 (2005). 


\section{FIGURE CAPTION}

Figure 1: reciprocal space map the (002) Bragg peak of (001) $\mathrm{SiC}$ recorded (a) along [110] and (b) along [1-10]. Reciprocal space map the (111) Bragg peak of (111) SiC recorded (a) along [2-1-1] and (b) along [11-2]. The streak labeled CTR is the crystal truncation rod. The streak labeled PSD is due to the transmittance function of the position sensitive detector. The remain streaks are due to stacking faults. $Q_{[h k l]}$ is the component of the scattering vector along a particular $[h k l]$ direction.

Figure 2: simulation of the diffuse intensity streaks with equations (1)-(3). Circles: experimental data; full line: simulation; dashed line: simulation with $\rho_{D}=0$. (a) Simulation of the [111] (lower curve) and [1-11] (upper curve) streaks. (b) Simulation of the [-111] (lower curve) and [11-1] (upper curve) streaks. $q_{<111>}$ is the deviation from the center of the Bragg peak along a particular $<111>$ streak. The curves are shifted vertically for clarity. 

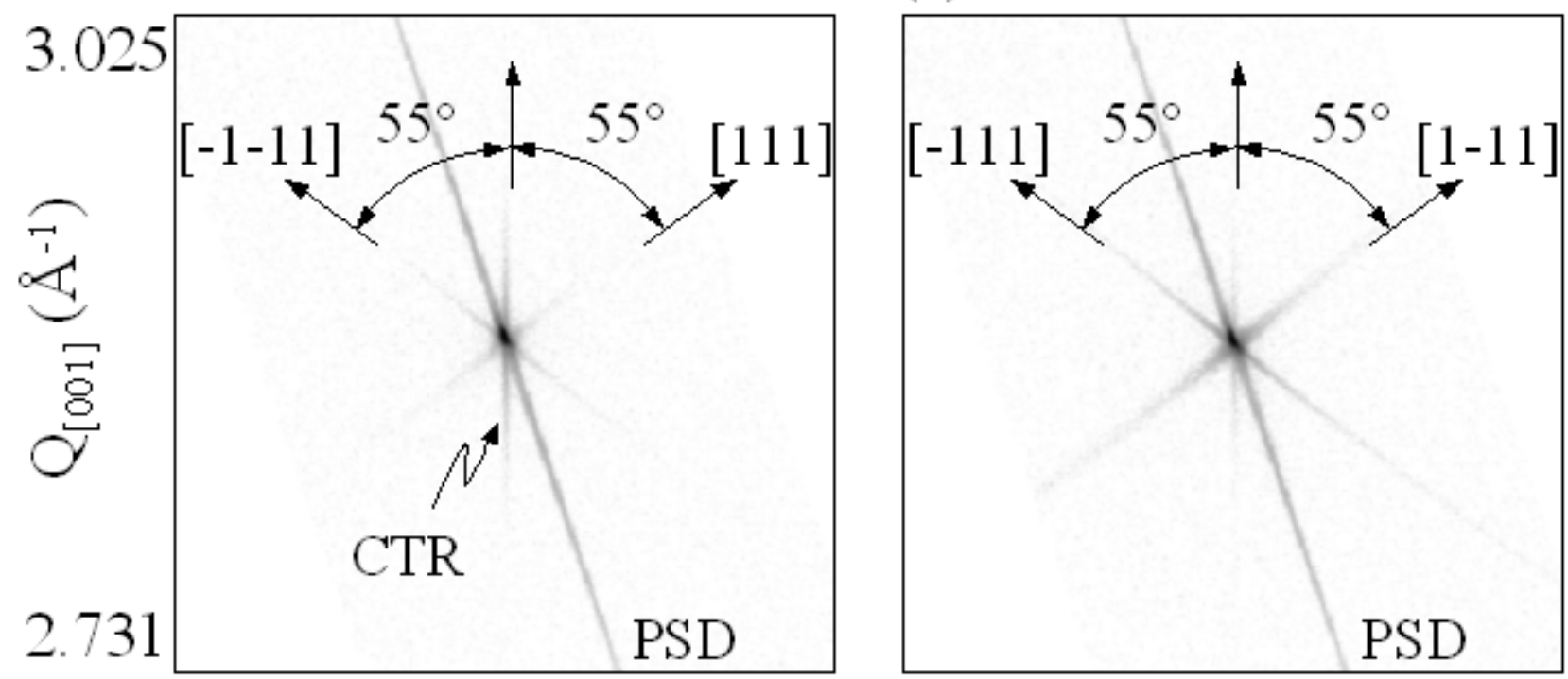

$-0.148$

$Q_{[110]}\left(\AA^{-1}\right)$

(c)

2.639

2.367

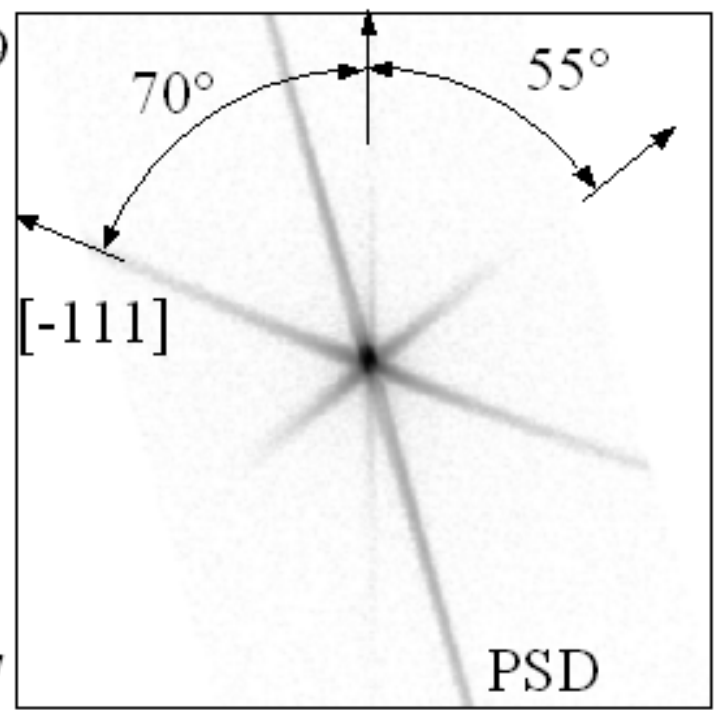

$0.148-0.148$

(d)

$Q_{[1-10]}\left(\AA^{-1}\right)$

0.148

$-0.136$

$0.136-0.136$
$\mathrm{Q}_{[2-1-1]}\left(\AA^{-1}\right)$
$Q_{[11-2]}\left(\AA^{-1}\right)$

PSD

0.136 

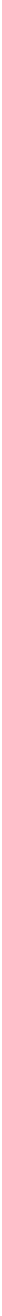